\title{
VANTAGENS DA CONSULTA PRÉ-OPERATÓRIA NA REABILITAÇÃO DA PESSOA SUBMETIDA A ARTROPLASTIA DA ANCA: REVISÃO INTEGRATIVA DA LITERATURA
}

\section{VENTAJAS DE LA CONSULTA PREOPERATORIA EN LA REHABILITACIÓN DE LA PERSONA SUJETA A ARTROPLASTIA DE CADERA: UNA REVISIÓN BIBLIOGRÁFICA}

\section{ADVANTAGES OF PREOPERATIVE CONSULTATION IN THE REHABILITATION OF THE PERSON SUBMITTED HIP ARTHROPLASTY: AN INTEGRATED LITERATURE REVIEW}

DOI 10.33194/rper.2020.v3.n1.5.5758 | Submetido 23.12.2019 | Aprovado 16/09/2020

\author{
Bruno Miguel Vivas Pina Pina ${ }^{1} \mathbb{D}$; Cristina Lavareda Baixinhoª \\ 1 - Centro Hospitalar Lisboa Norte; 2 - Escola Superior de Enfermagem de Lisboa
}

\section{RESUMO}

Introdução: A artroplastia da anca é uma cirurgia frequente que aliada à dor e à diminuição da mobilidade aumenta a dependência para o autocuidado e é preditora de perda de funcionalidade.

Objetivo: Determinar as vantagens da consulta de preparação pré-operatória na evolução da funcionalidade pósoperatória da pessoa submetida a artroplastia da anca.

Método: Revisão Integrativa da Literatura, foram definidos critérios de elegibilidade para a amostra bibliográfica.

Resultados: A amostra bibliográfica ficou constituída por 16 artigos que permitem responder ao objetivo da investigação. Os programas de recuperação estruturados, com consulta pré-operatória, diminuem as complicações pós-cirúrgicas e o tempo médio de internamento, promovem uma melhor gestão da dor e a recuperação mais rápida através de uma abordagem interprofissional.

Conclusões: 0 programa de reabilitação deve iniciar-se na fase pré-operatória porque o aumento do conhecimento sobre a cirurgia, programa de reabilitação e transição para a comunidade traduz-se em ganhos de saúde, sociais e económicos

Descritores: Enfermagem, Reabilitação, Capacitação, Artroplastia da anca, Consulta pré-operatória, Autocuidado

\section{RESUMEN}

Introducción: La artroplastia de la cadera es una cirugía frecuente que, combinada con dolor y disminución de la movilidad, aumenta la dependencia del cuidado personal y es un predictor de pérdida de funcionalidad.

Objetivo: determinar las ventajas de la consulta de preparación preoperatoria en la evolución de la funcionalidad postoperatoria de la persona sometida a artroplastia de cadera.

Método: Revisión Integral de Literatura, se definieron criterios de elegibilidad para la muestra bibliográfica.

Resultados: La muestra bibliográfica consistió en 16 artículos que nos permiten responder al objetivo de la investigación. Los programas de recuperación estructurados con consulta preoperatoria reducen las complicaciones postoperatorias y la duración promedio de la estadía, promueven un mejor manejo del dolor y una recuperación más rápida a través de un enfoque interprofesional. Conclusiones: El programa de rehabilitación debe comenzar en la fase preoperatoria porque un mayor conocimiento sobre cirugía, programa de rehabilitación y transición a la comunidad se traduce en beneficios para la salud, sociales y económicos.

Palabras clave: Enfermaría; Rehabilitación; Capacitación; Artroplastia de Reemplazo de Cadera; Autocuidado

\section{ABSTRACT}

Introduction: Hip arthroplasty is a frequent surgery that, combined with pain and decreased mobility, increases dependence on self-care and is a predictor of loss of functionality. Objective: To determine the advantages of preoperative preparation consultation in the evolution of postoperative functionality of the person undergoing hip artroplasty.

Method: Integrative Literature Review, eligibility criteria were defined for the bibliographic sample.

Results: The bibliographic sample consisted of 16 articles that allow us to respond to the research objective. Structured recovery programs with preoperative consultation reduce postoperative complications and average length of stay, promote better pain management and faster recovery through an interprofessional approach. 
Conclusions: The rehabilitation program should start in the preoperative phase because increased knowledge about surgery, rehabilitation program and transition to the community translates into health, social and economic gains.

Keywords: Nursing; Rehabilitation; Training; Arthroplasty (Hip replacement); Self Care

\section{INTRODUÇÃO}

O aumento da esperança média de vida da população e o consequente aumento das cirurgias ortopédicas, nomeadamente da artroplastia da anca, tem sido um ganho no campo da saúde, permitindo controlar a osteoartrose, a incapacidade e a dor secundária a esta. Todavia a cirurgia não é isenta de riscos. 0 tipo de cirurgia, a dor perioperatória, os défices funcionais (anteriores à cirurgia) e a diminuição da mobilidade no pós-operatório, podem traduzir-se, temporária ou definitivamente, em incapacidade, o que aliado a internamentos cada vez mais curtos, implica uma mudança de paradigma na prestação e organização dos cuidados a estas pessoas, com um enfoque claro na capacitação para a promoção do autocuidado(1).

Esta questão assume uma maior relevância quando as pessoas submetidas a artroplastia apresentam risco acrescido de fragilidade, imposto pelas comorbidades ou dependência prévia no autocuidado(1). A diminuição do tempo médio de internamento e o regresso atempado a casa, muitas vezes, sem a garantia da continuidade de cuidados de reabilitação aumenta o risco de perda de capacidade funcional após a cirurgia.

As pessoas são vulneráveis a experiências de perda de continuidade de cuidados, necessitando de apoio para continuar as orientações e desenvolverem competências específicas para manterem os cuidados iniciados no hospital ${ }^{(1-2)}$. A continuidade de cuidados garante a melhoria da qualidade dos mesmos e constitui uma estratégia adequada para uma política a seguir pelos diferentes serviços de saúde ${ }^{(2)}$.

Algumas instituições têm criado ou implementado programas estruturados para a capacitação, tanto para a pessoa submetida à cirurgia como do familiar cuidador e facilitar o regresso a casa. A existência de uma, ou mais, consultas pré-operatórias, com envolvimento do cuidador é importante para o esclarecimento de dúvidas e ensinos sobre a cirurgia, mas também para a compreensão e adesão ao programa de reabilitação( ${ }^{(3)}$. Todavia, muitos serviços de ortopedia continuam sem ter um programa de recuperação planeado e estruturado desde o pré ao pós-operatório, incluído a preparação do regresso a casa, contribuído para a perda da independência, do potencial de marcha e da capacidade de autocuidado $^{(1,4)}$. A ausência de programas de reabilitação aumenta o tempo de internamento e o tempo de recuperação, com impacto negativo na independência e capacidade funcional da pessoa ${ }^{(4,5)}$.

O Enfermeiro Especialista em Enfermagem de Reabilitação tem uma responsabilidade diferenciada não só na gestão do cuidado à pessoa com coxartrose, mas também na capacitação da sua família(5) e na prevenção da desfragmentação dos processos de reabilitação entre os níveis de cuidados (hospital e cuidados de saúde primários) ${ }^{(1)}$. Esta desfragmentação ou descontinuidade de cuidados especializados de reabilitação é pouco explorada na literatura, mas a diminuição do tempo médio de internamento e a contenção económica impõem uma articulação entre os EEER de ambos os contextos de cuidados de saúde, para garantir a segurança e a continuidade de cuidados à pessoa e ao seu familiar cuidador $^{(1)}$.

Tanto a questão da capacitação como da continuidade de cuidados são centrais nos programas estruturados de reabilitação da pessoa submetida a artroplastia da anca.

Os autores são consensuais a advogar que estes programas melhoram a recuperação pós-operatória, reduzem a ansiedade, fortalecem, a confiança da pessoa, melhoram a satisfação durante o internamento, reduzem custos, permitindo uma alta hospitalar mais rápida e em melhores condições ${ }^{(1,4,6)}$ e centrando o foco de cuidados no doente e na sua família(6).

Face ao exposto é objetivo desta Revisão Integrativa da Literatura (RIL) - Determinar as vantagens da consulta de preparação pré-operatória na evolução da funcionalidade pós-operatória da pessoa submetida a artroplastia da anca.

\section{MÉTODO}

O ponto de partida para o desenvolvimento da presente Revisão Integrativa da Literatura (RIL) foi a seguinte questão de investigação, elaborada de acordo com a mnemónica PEO (Population and their Problem; Exposure; Outcome and themes): "Quais as vantagens da consulta pré-operatória na reabilitação da pessoa submetida a artroplastia da anca?".

A opção por uma RIL justifica-se por o método possibilitar a síntese do conhecimento e a incorporação dos resultados na prática ${ }^{(7)}$. Na sua elaboração seguimos um protocolo com seis etapas: identificação do tema e seleção da hipótese ou questão de pesquisa, estabelecimento de critérios para inclusão e exclusão de estudos, definição das informações a serem extraídas, avaliação dos estudos incluídos, interpretação dos resultados e apresentação da revisão/síntese do conhecimento ${ }^{(8)}$.

A definição da pergunta possibilitou a definição dos critérios de elegibilidade dos artigos a incluir nesta RIL, com a finalidade de aumentar o rigor na pesquisa, delimitar o fenómeno em estudo e facilitar a identificação, leitura, extração e análise dos achados.

$\mathrm{Na}$ tabela 1 estão representados os critérios de inclusão para os estudos primários. 


\section{Critério de inclusão}

$\begin{array}{ll}\text { Participantes } & \begin{array}{l}\text { Pessoas adultas (com idade superior } \\ \text { e o seu }\end{array} \\ \begin{array}{l}\text { a } 18 \text { anos), de ambos os sexos, } \\ \text { problema }\end{array} & \text { submetidas a artroplastia da anca. }\end{array}$

Estudos primários de natureza qualitativa e/ou quantitativa, revisões sistemáticas da literatura, Tipo de estudo revisões integrativas da literatura, scoping review e protocolos ou normas de orientação clínica.

\begin{tabular}{l|l} 
Contexto & Internamento Hospitalar \\
$\begin{array}{l}\text { Fenómeno de } \\
\text { interesse }\end{array}$ & $\begin{array}{l}\text { Consulta pré-operatória em } \\
\text { programas estruturados de } \\
\text { reabilitação na artroplastia da anca. }\end{array}$
\end{tabular}

Tabela 1 - Critérios de inclusão dos estudos da RIL. Lisboa; 2018

A pesquisa efetuada foi realizada em Abril e Maio de 2018. Os descritores, usados em português, espanhol e inglês e em associações (AND e OR) foram: Orthopaedic patient (or orthopaedic chirurgic) AND Preoperative Education (or pre-operative self-efficacy education or patient education or education program) AND Hip Replacement (or joint replacement or arthroplasty).

A pesquisa foi efetuada nas bases de dados disponíveis nas plataformas da EBSCO, B-On, SCOPUS, ISI e JBI. O limite temporal estabelecido para a seleção dos artigos foi de 2013 a 2018, esta opção deveu-se ao estado de arte e à necessidade de identificar estudos recentes, dado que este tipo de cirurgia tem evoluído nos últimos, o tempo médio de internamento tem diminuído e por isso há variáveis nas pesquisas anteriores que não são sensíveis ao contexto atual.

A estratégia de pesquisa permitiu identificar 1258 artigos. A leitura do título permitiu eliminar 11 que estavam repetidos, a leitura do resumo possibilitou eliminar 1211, os restantes 36 artigos foram lidos na integra. Nesta fase foram eliminados 20 estudos: em 8 a amostra foram pessoas com artroplastia da anca e do joelho e os resultados não possibilitavam a avaliação das vantagens da consulta pré-operatória para as que foram submetidas a artroplastia da anca. Os restantes 12 artigos foram suprimidos porque não respondiam à questão de investigação. A amostra final ficou constituída por 16 artigos (Figura 1).

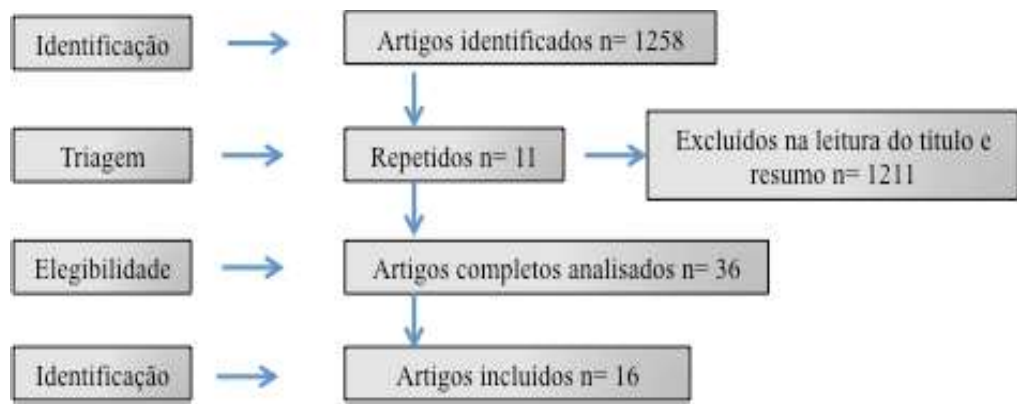

Figura1 - Fluxograma de seleção dos artigos para a RIL, elaborado a partir das recomendações PRISMA. Lisboa; 2018

Elaborou-se uma tabela com as informações a serem extraídas: artigo; autor(es), (ano de publicação), tipo de artigo; objetivo(s), métodos (se aplicável) e principais resultados.

\section{RESULTADOS}

Os 16 estudos que integram a amostra bibliográfica não são homogéneos, tem objetivos e desenhos diferentes, desde revisões, a estudos primários com abordagens quantitativas e qualitativas. Todavia as diferenças metodológicas a interpretação das fontes permitiu dar resposta à questão de investigação. No Quadro 1 apresentam-se os resultados extraídos dos artigos sobre as vantagens da consulta pré-operatória para as pessoas que são submetidas a artroplastia da anca.

\section{Artigo}

Pre-operative self-efficacy education vs. usual care for patients undergoing joint replacement surgery: a pilot randomized controlled trial

\section{Autores (ano)}

A educação pré-operatória encoraja as pessoas a assumir um papel central na sua recuperação promovendo resultados positivos a longo prazo.

Cooke et al. $(2016)^{(3)}$

Tal evidência encoraja à existência de diretrizes clínicas sobre a implementação de uma intervenção educativa específica no pré-operatório, assim como a promover cuidados de enfermagem de alta qualidade e melhorar os resultados nas pessoas.
Enhanced Recovery in Orthopedics: A Prospective Audit of an Enhanced Recovery Program for PatientsUndergoing Hip or Knee Arthroplasty

Total Hip Arthroplasty: Providing Preoperative Care for the Patient Undergoing
Brennan, Parsons $(2017)^{(4)}$

Programas de recuperação estruturados ajudam a reduzir o tempo de internamento, melhoram os resultados da cirurgia e aumentam a satisfação. 0 estudo salienta a melhor gestão da dor, redução de náuseas e vómitos, e recuperação mais rápida através de uma abordagem interprofissional.

Os cuidados pré-operatórios preparam a pessoa fisicamente; reduzem o risco de complicações, nomeadamente de infeções; permitem o despiste de infeções e a avaliação da condição física.

Schub, Caple $(2017)^{(9)} \quad$ Tem efeito positivo na redução da ansiedade.

Tem impacto social ao permitir a antecipação das necessidades de cuidados e apoio pós-operatório adequado no regresso a casa. 


\section{Artigo}

Autores (ano)

Resultados do Estudo

\begin{abstract}
Difference Between
Received and Expected Knowledge of Patients Undergoing Knee or Hip Replacement in Seven

European Countries
\end{abstract}

Education Attainment is Associated With Patientreported Outcomes: Findings From the Swedish Hip Arthroplasty Register

Klemetti et al. $(2015)^{(10)}$

Enhanced care for primary hip arthroplasty: factors affecting length of hospital stay $(2013)^{(12)}$

Greene et al. $(2014)^{(11)}$

A educação tem efeito positivo na funcionalidade. Os autores recomendam que a informação não deva incidir apenas nas questões físicas e funcionais, como é comum, recomendando uma abordagem mais holística e multidimensional.

Os profissionais de saúde necessitam de ensinar sobre dos riscos associados à cirurgia e ajuda a definir expectativas realistas no pós-operatório.

Os ensinos individualizados às características sociodemográficas e de escolaridade podem melhorar a satisfação da pessoa, diminuir a dor e os custos associados a este tipo de cirurgia.

Gerir a expectativa da pessoa e uma melhor preparação para a cirurgia é claramente um fator importante na redução do tempo médio de internamento.

A educação pré-operatória fornece a oportunidade de aquisição de conhecimentos, melhoria da capacidade de executar habilidades de autocuidado e reforça a capacidade de lidar com o experiência cirúrgica.

Patient Education: Teaching Heering, the Surgical Patient

Engelke $(2017)^{(13)}$

A educação pré-operatória é eficaz, consegue reduzir custos, diminuir o internamento pós-operatório no hospital e melhorar o conhecimento das habilidades de autocuidado, os comportamentos de autocuidado e a gestão de sinais e sintomas.

No major effects of preoperative education in patients undergoing hip or knee replacement - a systematic review

Aydin et al $(2015)^{(14)}$

Não houve nenhuma evidência convincente a favor da consulta préoperatória sobre a dor, o tempo médio de internamento, a satisfação, as complicações pós-operatórias, a mobilidade e as expectativas.

Há evidência do efeito da consulta na redução da ansiedade pré-operatória.

Jäppinen, Hämäläinen, A educação pré-operatória, leva a pessoa a adquirir conhecimentos, que

Patients' conceptions of preoperative physiotherapy education before hip arthroplasty

Kettunen,

Piirainen

$(2015)^{(15)}$ devem ser demonstrados com elementos práticos.

Isto requer uma relação de confiança entre a pessoa e o profissional de saúde, numa perspetiva de preparação para a reabilitação.

Conradsen, Gjerseth,

Patients' experiences from an education programme ahead of orthopaedic surgery - a qualitative study

O programa de educação pré-operatória promoveu a aceitação da cirurgia.

Para construir uma relação de confiança a educação pré-operatória deve ser em grupo e individual e deve ser realizada de forma a estimular e apoiar a confiança na equipa.

A informação deve ser realista e precisa.

\section{Preoperative Education for Hip and Knee Replacement: Never Stop Learning}

Pre-operative education prior to elective hip arthroplasty surgery improves postoperative outcome
Edwards, Mears, Barnes $(2017)^{(17)}$

Moulton,

Evans, Starks \& Smith $(2016)^{(18)}$
As aulas de educação pré-operatória são um elemento essencial para uma participação bem-sucedida da pessoa no processo de recuperação.
The effectiveness of orthopedic patient education in improving patient outcomes: a systematic review protocol

The perioperative dialogue - a model of caring for the patient undergoing a hip or a knee replacement surgery under spinal anaesthesia
Majid, Lee, Plummer $(2015)^{(19)}$

Pulkkinen, Junttila, Lindwall $(2016)^{(20)}$
A educação pré-operatória produz internamentos mais curtos e redução de custos. Há também efeitos sobre os scores de mobilização, que melhoram no pós-operatório.
Resultados significativos ao nível da diminuição do $\mathrm{n} .{ }^{\circ}$ de dias de internamento, das pessoas que receberam educação pré-operatória, comparada as pessoas que não receberam qualquer tipo de educação ou informações.
A preparação pré-operatório estruturada permite criar maior empatia entre a pessoa e os profissionais e vai de encontro dos desejos de cada indivíduo, garantindo assim uma maior qualidade dos cuidados prestados. 


\section{Artigo}

Autores (ano)

Resultados do Estudo

\begin{tabular}{|c|c|c|}
\hline $\begin{array}{l}\text { Unfulfilled Expectations } \\
\text { After Total Hip and Knee } \\
\text { Arthroplasty Surgery: There } \\
\text { Is a Need for Better } \\
\text { Preoperative Patient }\end{array}$ & $\begin{array}{l}\text { Tilbury et al. } \\
(2016)^{(21)}\end{array}$ & $\begin{array}{l}\text { Ajuda na gestão das expectativas peri-operatórias, que foram satisfeitas ou } \\
\text { mesmo ultrapassadas com a consulta pré operatória. Efeito no controlo da } \\
\text { dor e na promoção da função. }\end{array}$ \\
\hline $\begin{array}{l}\text { Preoperative education for } \\
\text { hip or knee replacement } \\
\text { (Review) }\end{array}$ & $\begin{array}{l}\text { McDonald, } \\
\text { Page, Beringer, } \\
\text { Wasiak, } \\
\text { Sprowson } \\
(2014)^{(22)}\end{array}$ & $\begin{array}{l}\text { A educação pré-operatória pode representar um cuidado adjuvante, com } \\
\text { baixo risco de efeitos indesejáveis, particularmente em certas pessoas, com } \\
\text { depressão, ansiedade ou expectativas irrealistas. }\end{array}$ \\
\hline
\end{tabular}

Quadro 1 - Amostra bibliográfica da RIL. Lisboa, 2018

\section{DISCUSSÃO}

Os estudos desta pesquisa são heterogéneos, com desenhos de estudo diferentes, uns qualitativos, outros quantitativos. Há diferenças consideráveis em termos de amostra, intervenção implementada, conceitos usados, medidas e momentos de avaliação da eficácia da consulta e do seu impacto na funcionalidade da pessoa submetida a artroplastia. Os instrumentos para avaliação da evolução da funcionalidade são dispares, alguns não estão validados para a população portuguesa, o que dificulta a comparação e a discussão dos resultados.

Os achados, desta revisão, vão de encontro à literatura sobre o tema, que refere como benefícios da consulta pré-operatória: a diminuição da ansiedade, do stress, da dor e do tempo médio de internamento (2,5 dias-3,4 dias) ${ }^{(4,9,21,23-24)}$; a promoção da independência para o autocuidado e para a realização das atividades de vida diária) ${ }^{(25-26)}$; garantido a autonomia da pessoa e da sua família durante a prestação de cuidados $^{(1,23)}$.

Atualmente existem programas específicos para a recuperação e reabilitação da pessoa submetida a artroplastia total da anaca, como o programa Rapid Recovery, que tem enorme consenso a nível internacional pelo impacto na recuperação e reabilitação das pessoas submetidas a cirurgia. Este tem como principal fundamento fazer com que a pessoa seja participante ativa na sua reabilitação, através da educação e preparação pré-operatória, otimizando os recursos e capacidades individuais, tendo por base uma abordagem multidisciplinar, envolvendo ao longo do mesmo cirurgiões, anestesistas, enfermeiros generalistas e especialistas em reabilitação, fisioterapeutas, farmacêuticos e até mesmo pessoas que foram anteriormente sujeitas à mesma cirurgia(27). O programa reduz a ansiedade, fortalece a confiança da pessoa, melhora a satisfação do doente durante o internamento, reduz custos para o hospital, permitindo uma alta hospitalar mais rápida ${ }^{(13,27)}$.

O facto de ser efetuada uma a três semanas antes da cirurgia possibilita o despiste de infeções e a avaliação da condição física pré-cirurgia ${ }^{(9)}$, o que contribui para o aumento da segurança operatória e permite individualizar o programa de reabilitação em função da mobilidade, força muscular e amplitude articular préoperatória.
A educação pré-operatória fornece não só a oportunidade de aquisição de conhecimentos sobre a cirurgia e implicações no autocuidado, após a colocação da prótese, mas também a possibilidade de ensinar e treinar habilidades, em segurança, prevenindo movimentos luxantes durante a realização de atividades de vida diária( ${ }^{(9)}$. Simultaneamente, ajuda na gestão das expectativas peri-operatórias(21). Porém, para atingir a sua finalidade os ensinos têm de ser individualizados às características sociodemográficas e de escolaridade da população alvo ${ }^{(11)}$.

Os autores reforçam ainda a importância da consulta para aumento da adesão ao programa de reabilitação peri-operatório e advogam que a conjugação da consulta com uma intervenção adequada de reabilitação contribui para o aumento da independência funcional no momento da alta ${ }^{(1,24)}$, diminui as complicações associadas ao internamento e os reinternamentos, até pelo facto de contribuir para comportamentos de autocuidado seguros e para a gestão de sinais e sintomas ${ }^{(1,13)}$.

Por outro lado, a preparação antes do internamento para cirurgia, com inclusão de um programa de reabilitação motora e respiratória, prepara a pessoa fisicamente para a cirurgia e programa de reabilitação após a artroplastia $^{(9)}$, garantindo ganhos funcionais, com a mobilização, levante nas primeiras 24 horas após a cirurgia e treino de marcha precoce, o que promove ganhos na execução das atividades de vida diária, dado que a marcha é o autocuidado que mais influencia os outros autocuidados ${ }^{(28)}$.

$\mathrm{Na}$ revisão da literatura emerge com um beneficio da consulta pré-operatória a influência positiva na experiência de internamento(13), este é um indicador, usualmente, descurado e não mensurado pela investigação. Os autores acrescem que a consulta e organização dos cuidados melhora a experiência de internamento e permite lidar melhor com a cirurgia, contribuindo para a satisfação com os cuidados $^{(4,13)}$. Motivo pelo qual os profissionais de saúde devem estender a educação pré-operatória aos riscos associados à cirurgia e ajudar a definir expectativas realistas no pós-operatório ${ }^{(11)}$.

A gestão da expectativa da pessoa e uma boa preparação para a cirurgia são fatores majores para a redução do tempo de internamento ${ }^{(12)}$. No entanto, 
numa das pesquisas que integram esta RIL os investigadores não encontraram evidência convincente a favor da consulta pré-operatória sobre a dor, o tempo médio de internamento, a satisfação, as complicações pós-operatórias, a mobilidade e as expectativas ${ }^{(14)}$. Os autores só referem evidência no efeito da consulta na redução da ansiedade pré-operatória ${ }^{(14)}$.

Face ao exposto é possível confirmar os resultados dos estudos que advogam como grande vantagem da consulta a redução dos custos ${ }^{(11,13)}$. A educação préoperatória pode representar um cuidados adjuvante, com baixo risco de efeitos indesejáveis, particularmente em pessoas, com depressão, ansiedade ou expectativas irrealistas ${ }^{(22)}$.

De salientar ainda o elevado valor social deste tipo de programas ao permitir a antecipação das necessidades e apoio adequado após a alta, no momento do regresso a casa ${ }^{(9)}$ e a necessidade de desenvolver uma relação de confiança entre a pessoa e o profissional de saúde, numa perspetiva de preparação para a reabilitação(15).

\section{Limitações do estudo}

Para além das restrições temporais foram introduzidos outros filtros na pesquisa efetuada, nomeadamente: Full Text; Idiomas Português, Espanhol e Inglês, o que pode ter limitado a obtenção de outros artigos que enriquecessem esta revisão.

\section{CONCLUSÃO}

A consulta pré-operatória associada a programas estruturados, multiprofissionais, de reabilitação iniciados ainda na fase pré-operatória, tem vantagens, nomeadamente sobre a gestão eficaz da dor, da angústia e da ansiedade; aumenta os conhecimentos sobre a cirurgia e alterações no autocuidado, diminui o tempo médio de internamento com ganhos económicos e melhor aceitação e adesão ao programa de reabilitação individualizado pré-estabelecido.

De referir que a falta destes programas leva a dificuldades desde a admissão até à falta do planeamento da alta, com impacto negativo no autocuidado e na dependência/independência no momento do regresso a casa. A falta de informação e de organização dos cuidados, em torno do processo de reabilitação da pessoa e sua família pode levar a que o internamento seja vivido com dúvidas e desconhecimento do programa de reabilitação, condicionando que no momento da alta a pessoa esteja em piores condições funcionais do que antes da cirurgia.

\section{REFERÊNCIAS BIBLIOGRÁFICAS}

1. Ferreira, E.M., Lourenço, O.M., Costa, P.V., et al. Active Life: a project for a safe hospital-community transition after arthroplasty. Rev Bras Enferm, 2019;72(1):147-153. doi: https://dx.doi.org/10.1590/0034-7167-2018-0615

2. Mendes F, Gemito MLP, Parreirinha C, Cladeira EC, Serra IC, CasasNovas MV. Continuity of care from the perspective of users. Cienc Saúde Coletiva. 2017; 22(3):843-55. doi: https: / /dx.doi.org/10.1590/1413-81232017223.26292015
3. Cooke M, Walker R, Aitker L, Freeman A, Pavey S, Cantrill R. Preoperative self-efficacy education vs. usual care for patients undergoing joint replacement surgery: a pilot randomised controlled trial. Scand J Caringg Sci. 2016; 30(1):74-82 doi:10.1111/scs.12223

4. Brennan C, Parsons G. Enhanced Recovery in Orthopedics: A Prospective Audit of an Enhanced Recovery Program for Patients Undergoing. Hip or Knee Arthroplasty. Medsurg Nurs. 2017;26(2):99$104 . \quad$ Available

https://www.ncbi.nlm.nih.gov/pubmed/30304589

from:

5. Pereira SK, Santana RF, Morais VSC, Soares TS, Silva DM. Discharge planning in post-operative of elderly: multiple cases study. Rev Fund Care Online. 2016;8(4),4949-55. doi: https: / /dx.doi.org/10.9789/2175-5361.2016.v8i4.4949-4955

6. Aydin D, Klit J, Jacobsem S, Troelsen A, Husted H. No major effects of preoperative education in patients undergoing hip or knee replacement - a systematic review. Dan Med J 2015;62(7):A5106. Available from: https://ugeskriftet.dk/dmj/no-major-effectspreoperative-education-patients-undergoing-hip-or-kneereplacement-systematic

7. Sousa LMM, Marques-Vieira CMA, Severino SSP, Antunes AV. A metodologia de revisão integrativa da literatura em enfermagem. Revista Investigação em Enfermagem. 2017; Ser.II(21):17-26. Available from: https://repositoriocientifico.essatla.pt/bitstream/20.500.12253/1311/1/Metodologia\% 20de\%20Revis\%C3\%A3o\%20Integrativa_RIE21_17-26.pdf

8. Mendes KDS, Silveira RCCP, Galvão CM. Revisão integrativa: método de pesquisa para a incorporação de evidências na saúde e na enfermagem. Texto Contexto Enferm. 2008;17(4):758-64. doi: http://dx.doi.org/10.1590/S0104-07072008000400018.

9. Schub T, Caple C. Total Hip Arthroplasty: Providing Preoperative Care for the Patient Undergoing. Cinahl Information Systems. 2017. Avaialble from: Nursing Reference Center

10. Klemetti S, Leino-Kilpi, Cabrera E, et al. Difference Between Received and Expected Knowledge of Patients Undergoing Knee or Hip Replacement in Seven European Countries. Clin Nurs Res, 2015;24(6): 624-643. doi: 10.1177/1054773814549992. Epub 2014 Sep 17.

11. Greene M, Rolfson O, Nemes S, Gordon M, Malchau H, Garellick G. Education Attainment is Associated With Patient-reported Outcomes: Findings From the Swedish Hip Arthroplasty Register. Clin Orthop Relat Res. 2014;472(6):1868-76. doi: 10.1007/s11999-0143504-2. Epub 2014 Feb 19.

12. Panteli M, Habeeb S, McRoberts J, Porteous M. (Enhanced care for primary hip arthroplasty: factors affecting length of hospital stay. Eur J Orthop Surg Traumatol. 2014;24:353-358. doi: 10.1007/s00590013-1188-z.

13. Heering $H$, Engelke $Z$. Patient Education: Teaching the Surgical Patient. Cinahl Information Systems. 2017. Avaialble from: Nursing Reference Center

14. Aydin D, Klit J, Jacobsem S, Troelsen A, Husted H. No major effects of preoperative education in patients undergoing hip or knee replacement - a systematic review. Dan Med J. 2015; 62 ${ }^{(7)}$. pii: A5106. Avaialble from: https://ugeskriftet.dk/dmj/no-major-effectspreoperative-education-patients-undergoing-hip-or-kneereplacement-systematic

15. Jäppinen A, Hämäläinen $H$, Kettunen $T$, Piirainen A. Patients' conceptions of preoperative physiotherapy education before hip arthroplasty. Eur J Physiother. 2015;17:148-57. Doi: 10.3109/21679169.2015.1061051

16. Conradsen S, Gjerseth M, Kvangarsnes M. Patients' experiences from an education programme ahead of orthopaedic surgery - a qualitative study. J Clinic Nurs. 2016;25 (19-20): 2798-806. doi: 10.1111/jocn.13281. Epub 2016 Apr 8.

17. Edwards P, Mears S, Barnes C. Preoperative Education for Hip and Knee Replacement: Never Stop Learning. Curr Rev Musculoskelet Med. 2017;10(3):356-64. doi: 10.1007/s12178-017-9417-4.

18. Moulton L, Evans PA, Starks I, Smith T. Pre-operative education prior to elective hip arthroplasty surgery improves postoperative outcome. Int Orthop. 2015;39(8):1483-6. doi: 10.1007/s00264-0152754-2. Epub 2015 Apr 11.

19. Majid N, Lee S, Plummer V. The effectiveness of orthopedic patient education in improving patient outcomes: a systematic review protocol. JBI Database System Rev Implement Rep. 2015;13(1):12233. doi: 10.11124/jbisrir-2015-1950.

20. Pulkkinen M, Junttila K, Lindwall L. Preoperative education for hip or knee replacement (Review). Scand J Caring Sci. 2016;30(1): 145-53. doi: 10.1111/scs.12233. Epub 2015 Apr 28. 
21. Tilbury C, Haanstra TM, Leichtenberg CS, et al. Unfulfilled Expectations After Total Hip and Knee Arthroplasty Surgery: There Is a Need for Better Preoperative Patient Information and Education. J Arthroplasty. 2016;31(10):2139-45. doi: 10.1016/j.arth.2016.02.061. Epub 2016 Mar 17.

22. McDonald S, Page MJ, Beringer K, Wasiak J, Sprowson A. Preoperative education for hip or knee replacement. Cochrane Database Syst Rev. 2014. Issue 5. 13;(5):CD003526. doi: 10.1002/14651858.CD003526. pub3.

23. Duarte VDS, Santos MLD, Rodrigues KDA, Ramires JB, Arêas GPT, Borges GF. Exercícios físicos e osteoartrose: uma revisão sistemática.

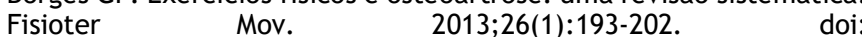
http://dx.doi.org/10.1590/S0103-51502013000100022.

24. Yoon RS, Nellans KW, Geller JA, Kim AD, Jacobs MR, Macaulay W. Patient Education Before Hip or Knee Arthroplasty Lowers Length of Stay. J Arthroplasty. 2010; 25(4):547-51. doi: 10.1016/j.arth.2009.03.012. Epub 2009 May 8.

25. Violante $P$, Cruz A. Efetividade de ensino pré-operatório em doentes submetidos a artroplastia total da anca. Série Monográfica Educação e Investigação em Saúde Enfermagem de Reabilitação: Resultados de Investigação. Coimbra: Unidade de Investigação em Ciências da Saúde: Enfermagem. 2016, p.43-63.

26. Desmeules F, Hall J, Woodhouse LJ Prehabilitation Improves Physical Function of Individuals with Severe Disability from Hip or Knee Osteoarthritis. Physiother Can. 2014;65(2):116-24. doi: 10.3138/ptc.2011-60.

27. Akhtar K, Burne D. Optimization of the patient undergoing total knee arthroplasty - the rapid recovery program. JCRMM. 2010;1(2): 1 . $4 . \quad$ Available from: https: //pdfs. semanticscholar.org/2177/bc4ba9b481ef729684335118 1082a0716053.pdf?_ga $=2.151661881 .1241756114 .1577113325$ -

1538352486.1568649449

28. Baixinho CL. Outcome after femoral neck fracture. Rev Baiana Enferm. 2011;25(3):311-9.

http://dx.doi.org/10.18471/rbe.v25i3.5480 